\section{(6) OPEN ACCESS}

\title{
Clinical features of HLA-B27-positive acute anterior uveitis with or without ankylosing spondylitis in a Chinese cohort
}

\author{
Peizeng Yang, ${ }^{1}$ Wenjuan Wan, ${ }^{1}$ Liping Du, ${ }^{1}$ Qingyun Zhou, ${ }^{1}$ Jian Qii, Liang Liang, ${ }^{1}$ \\ Chaokui Wang, ${ }^{1}$ Lili Wu, ${ }^{1}$ Aize Kijlstra ${ }^{2}$
}

${ }^{1}$ The First Affiliated Hospital of Chongqing Medical University, Chongqing Key Laboratory of Ophthalmology and Chongqing Eye Institute, Chongqing, China ${ }^{2}$ University Eye Clinic Maastricht, Maastricht, The Netherlands

\section{Correspondence to} Dr Peizeng Yang, Department of Ophthalmology, 1 You Yi Road, Yu Zhong District, Chongqing 400016, China; peizengycmu@ 126.com

Received 12 August 2016 Revised 21 April 2017 Accepted 19 May 2017 Published Online First 12 June 2017

\section{Check for updates}

To cite: Yang $\mathrm{P}$, Wan W, Du L, et al. Br J Ophthalmol 2018;102:215-219.

\section{ABSTRACT}

Aims To characterise the clinical features of human leucocyte antigen (HLA)-B27 ${ }^{+}$acute anterior uveitis (AAU) patients with or without ankylosing spondylitis (AS) and investigate the retinal vascular involvement in these patients.

Methods A total of $1056 \mathrm{HLA}-\mathrm{B} 27^{+}$AAU patients (1525 eyes) were retrospectively studied from April 2008 to February 2016. Patients were divided into human leucocyte antigen (HLA)-B27 ${ }^{+} \mathrm{AS}^{+}$and $\mathrm{HLA}-\mathrm{B} 27^{+} \mathrm{AS}^{-}$ group. Clinical features including the onset of uveitis, laterality, the age at first attack, clinical examinations, best corrected visual acuity (BCVA), abnormalities in fundus fluorescence angiography (FFA) and complications were determined and compared between these two groups.

Results There were 581 (55.0\%) and 475 (45.0\%) patients respectively classified into $\mathrm{HLA}-\mathrm{B} 27^{+} \mathrm{AS}^{+}$and HLA-B $27^{+} \mathrm{AS}^{-}$group. Males had a higher prevalence than females in the HLA-B27 ${ }^{+} \mathrm{AS}^{+}$group $(75.2 \%)$ as compared with the HLA-B27 ${ }^{+} \mathrm{AS}^{-}$group $(51.8 \%$, $\mathrm{p}<0.001)$. The $\mathrm{HLA}-\mathrm{B} 27^{+} \mathrm{AS} \mathrm{S}^{+}$patients showed a higher percentage of bilateral/alternating involvement $(47.3 \%)$ as compared with the HLA-B27 ${ }^{+} \mathrm{AS}^{-}$group (36.6\%, $\mathrm{p}=0.001$ ). A higher percentage of fibrinous exudation, synechiae as well as complications including complicated cataract and secondary glaucoma were found in the HLA-B27 ${ }^{+} \mathrm{AS}^{+}$group as compared with the HLA$B 27^{+} \mathrm{AS}^{-}$group. Worse visual outcome as indicated by a higher percentage of patients with $B C V A<0.5$ and with $\mathrm{BCVA}<0.05$ was noted in the HLA-B27 ${ }^{+} \mathrm{AS}^{+}$group as compared with the $\mathrm{HLA}-\mathrm{B}^{2} \mathrm{7}^{+} \mathrm{AS}^{-}$group both before and after treatment. FFA showed mild capillary fluorescence leakage in the late phase with indistinctly defined margins on the peripheral retina in $39.3 \%$ of $\mathrm{HLA}-\mathrm{B} 27^{+}$ AAU patients. There was no difference concerning the retinal vascular involvement between these two groups. Conclusions Our study confirmed that HLA-B27 ${ }^{+} \mathrm{AS}^{+}$ patients show a higher percentage of males, more common bilateral involvement, a higher frequency of fibrinous exudates, synechiae and secondary glaucoma as compared with $\mathrm{HLA}-\mathrm{B} 2 \mathrm{7}^{+} \mathrm{AS}^{-}$patients. Visual outcome was poorer, possibly due to the higher prevalence of complicated cataract in $\mathrm{HLA}-\mathrm{B} 27^{+} \mathrm{AS}^{+}$patients. Retinal vascular involvement was not uncommon in HLA-B27 ${ }^{+}$ AAU patients.

\section{INTRODUCTION}

Acute anterior uveitis (AAU) is the most common uveitis entity worldwide and is characterised by inflammation of the anterior chamber lasting no longer than 3 months. The genetic marker human leucocyte antigen (HLA)-B27 is strongly associated with AAU, whereby the percentage of HLA-B27 positivity among AAU patients varies between $12 \%$ and $88 \%{ }^{1-4}$ It has been reported that AAU associated with HLA-B27 accounts for up to $81 \%$ of AAU cases in Taiwan, ${ }^{5}$ a frequency that is much higher than that reported from many other countries. Earlier reports from our group in mainland China found that $63 \%$ of AAU patients were HLA-B27 $7^{+}{ }^{6}$

HLA-B2 $7^{+}$AAU is frequently associated with seronegative spondyloarthropathies, such as ankylosing spondylitis (AS), inflammatory bowel disease, psoriatic arthritis and reactive arthritis. ${ }^{7}$ Although seronegative spondyloarthropathies share certain clinical characteristics, the pattern of ocular involvement is somewhat different for each entity. ${ }^{8}$ AS is considered to be the major systemic disease presentation $(55 \%-90 \%)^{910}$ in HLA-B27 $7^{+}$AAU.

Although descriptions of HLA-B27 ${ }^{+} \mathrm{AS}^{+}$AAU have been published previously, ${ }^{11}$ it is not yet clear how the occurrence of AS affects the manifestations of HLA-B $27^{+}$AAU, especially in Chinese patients. In this study, we therefore examined a large cohort of $\mathrm{HLA}-\mathrm{B} 27^{+}$AAU patients associated with or without AS to evaluate the clinical features, visual prognosis as well as retinal vascular involvement

\section{MATERIALS AND METHODS}

A retrospective cohort study was performed at the uveitis clinic of the First Affiliated Hospital of Chongqing Medical University. Consecutive patients with AAU observed from April 2008 to February 2016 were included in this study. The study was authorised by the institutional review board of the First Affiliated Hospital of Chongqing Medical University, Chongqing, China.

AAU was defined as the presence of inflammatory cells in the anterior chamber and dust-like keratic precipitates with aqueous flare, absence of posterior vitreous cells and other features of posterior segment inflammation apart from macular and optic disc oedema. The intraocular inflammation was characterised by a sudden onset with a duration of less than 3 months. ${ }^{12}$

In this study, we only compared the differences of the clinical manifestation between HLA-B2 $27^{+} \mathrm{AS}^{+}$ AAU patients and HLA-B $27^{+} \mathrm{AS}^{-}$AAU patients. Therefore, patients were excluded if (1) the HLA test or sacroiliac joint radiographs were not available, (2) 
they had a follow-up less than 3 months, (3) they had a viral (herpes simplex virus (HSV)1, HSV2, varicella-zoster virus, Epstein-Barr virus, cytomegalovirus) or bacterial (luetic or tubercular) aetiology, (4) the patients with other uveitis entities such as Behcet's disease or Vogt-Koyanagi-Harada syndrome or sarcoidosis and (5) the AAU was accompanied with other spondyloarthropathies such as inflammatory bowel disease, psoriatic arthritis, reactive arthritis and sarcoidosis since the numbers of such cases was quite small. AS was diagnosed by rheumatologists according to the Modified New York Criteria. ${ }^{13}$ The radiological examinations of the sacroiliac joint included radiographs, CT or MRI and were evaluated by specialists from our rheumatology or orthopaedics department.

The onset of uveitis, laterality, the age at first attack, disease course since first episode of AAU, as well as inflammatory back pain or morning stiffness, release position and other general history parameters were recorded. A complete ophthalmic evaluation including best corrected visual acuity (BCVA), slit-lamp examination of the anterior and posterior segment, intraocular pressure (IOP) and fundus examination after pupillary dilation was performed. Fundus fluorescence angiography (FFA) was non-selectively performed. FFA was also confirmed by clinical manifestation and optical coherence tomography (OCT) findings. FFA was not performed in case of opacity in the ocular refractive pathway, renal or cardiac disease or hypersensitivity to fluorescein. Blood testing (complete blood count, HLA-B27 test) were routinely included. Rheumatological examination, chest and sacroiliac radiographs, CT or MRI were obtained for each patient. Disease activity of AS was also evaluated using the Bath Ankylosing Spondylitis Disease Activity Index (BASDAI) (range 0-10), ${ }^{14}$ and Bath Ankylosing Spondylitis Functional Index (BASFI) (range 0-10). ${ }^{15}$

AAU was treated with topical steroids combined with cycloplegic eye-drops. In a few patients with massive fibrous exudates in the anterior chamber or hypopyon, oral corticosteroids (usually $20 \mathrm{mg}$ prednisone per day as initial dose) were also used for 3 to 4 weeks. Subconjunctival injection of triamcinolone was performed in patients with contraindications such as increased blood glucose level, severe gastroduodenal ulcer or osteoporosis. The patients with AS were also treated with systemic low-dose corticosteroids (usually $20 \mathrm{mg} /$ day) combined with ciclosporin (generally $3 \mathrm{mg} / \mathrm{kg} / \mathrm{day}$ ) or recommended to be treated by specialists from our rheumatology or orthopaedics department.

\section{Statistical analysis}

Data analysis was performed using SPSS V.16.0 (SPSS). The Kruskal-Wallis test was used to compare variables. The Wilcoxon matched pair test was used to compare paired variables. Categorical variables were analysed using Pearson $\chi^{2}$ test with Fisher's exact test. Pearson and Spearman tests were applied for the correlational analyses. $\mathrm{p}$ Values lower than 0.05 were considered statistically significant.
RESULTS

\section{General information}

A total of 11698 uveitis patients, including $1567 \mathrm{HLA}^{-B} 27^{+}$and 983 HLA-B27 ${ }^{-}$AAU patients, were referred to our uveitis clinic during the study period. We included 1056 (1525 eyes) HLA-B27 ${ }^{+}$patients that matched our inclusion criteria and divided them into two groups according to rheumatological results. Five hundred and eighty-one (55.0\%) and 475 (45.0\%) patients were respectively classified into the HLA-B $27^{+} \mathrm{AS}^{+}$group and HLA-B $27^{+} \mathrm{AS}^{-}$group (table 1). All of the investigated patients were followed from 3 months to 72 months, with a mean follow-up of $23.7 \pm 9.5$ months. Average BASFI and BASDAI scores of AAU patients with AS was $1.8 \pm 1.7$ and $2.4 \pm 1.6$ respectively at their first visit in our uveitis clinic.

\section{Sex, laterality and onset age}

Males (75.2\%) showed a higher prevalence than females in the HLA-B $27^{+} \mathrm{AS}^{+}$group as compared with the HLA-B $27^{+} \mathrm{AS}^{-}$ group $(51.8 \%, \mathrm{p}<0.001)$ (table 1$)$.

Both unilateral and bilateral involvement was observed in HLA-B $27^{+}$AAU patients. A total of 348 patients presented as monocular attacks of AAU, alternating between eyes during recurrences, which was also defined as bilateral involvement. The HLA-B $27^{+} \mathrm{AS}^{+}$patients showed a higher percentage of bilateral/alternating involvement (47.3\%) as compared with the HLA-B27 ${ }^{+}$AS $^{-}$group $(36.6 \%, p=0.001)$ (table 1 ).

The onset age of AAU was $33.5 \pm 10.9$ years in the HLA-B $27^{+} \mathrm{AS}^{+}$group and was exactly the same as in the HLA-B27 ${ }^{+} \mathrm{AS}^{-}$group $(33.5 \pm 11.3$ years; $\mathrm{p}=0.550)$.

\section{Clinical features}

The occurrence of fibrinous exudation and synechiae was higher in the HLA-B2 $7^{+} \mathrm{AS}^{+}$group as compared with the HLA-B27 ${ }^{+} \mathrm{AS}^{-}$ group (table 2). The percentage of other clinical manifestations such as corneal endothelium wrinkling and hypopyon were not significantly different between the two groups.

\section{Results of FFA}

FFA was non-selectively performed in 903 eyes of 648 patients, which included 466 eyes of 331 patients in the HLA-B27 ${ }^{+} \mathrm{AS}^{+}$ group and 437 eyes of 317 patients in the HLA-B27 ${ }^{+} \mathrm{AS}^{-}$group.

FFA showed vascular leakage in the late phase in the peripheral retina (figure 1). This manifestation was found in $39.3 \%$ of all HLA-B27 $7^{+}$AAU patients, with a percentage of $36.5 \%$ in the HLA-B $27^{+} \mathrm{AS}^{+}$group and $42.3 \%$ in the HLA-B $27^{+} \mathrm{AS}^{-}$group. Vascular leakage mostly disappeared in about 4 to 6 weeks after disease onset without any other fundus complications (figure 1). Cystoid macular oedema (1.7\%) and optic disc hyperfluorescence $(5.4 \%)$ were also found in the affected eyes of certain patients.

Table 1 Demographic data of study subjects

\begin{tabular}{lllll}
\hline & AS+ & AS $^{-}$ & p Value & Total \\
\hline AAU patients & 581 & 475 & N/A & 1056 \\
$\quad$ Male & $437(75.2 \%)$ & $246(51.8 \%)$ & $<0.001^{* * *}$ & 683 \\
$\quad$ Female & $144(24.8 \%)$ & $229(48.2 \%)$ & & 373 \\
Eyes studied & 883 & 642 & N/A & 1525 \\
OD/OS & $432 / 451$ & $332 / 310$ & $0.001^{* *}$ & $764 / 761$ \\
Bilateral involvement & $275(47.3 \%)$ & $174(36.6 \%)$ & 449 & 607 \\
Unilateral involvement & $306(52.7 \%)$ & $301(63.4 \%)$ & & \\
\hline
\end{tabular}

${ }^{*} \mathrm{p}<0.05,{ }^{* *} 0.001 \leq \mathrm{p} \leq 0.01,{ }^{* * *} \mathrm{p}<0.001$

$\mathrm{AAU}$, acute anterior uveitis; $\mathrm{AS}$, ankylosing spondylitis; $\mathrm{OD} / \mathrm{OS}$, oculus dexter/oculus sinister; $N / A$, not applicable. 
Table 2 Comparison of clinical features between $\mathrm{HLA}-\mathrm{B} 27^{+} \mathrm{AS}^{+}$ group and $\mathrm{HLA}-\mathrm{B} 27^{+} \mathrm{AS}^{-}$group

\begin{tabular}{|c|c|c|c|c|c|}
\hline \multirow[b]{2}{*}{ Clinical characteristic } & \multicolumn{2}{|l|}{$\begin{array}{l}\mathrm{AS}^{+} \\
(\mathrm{n}=883)\end{array}$} & \multicolumn{2}{|l|}{$\begin{array}{l}\mathrm{AS}^{-} \\
(\mathrm{n}=642)\end{array}$} & \multirow[b]{2}{*}{$\mathrm{p}$ Value } \\
\hline & Eyes $(n)$ & Rate $\%$ & Eyes $(n)$ & Rate $\%$ & \\
\hline $\begin{array}{l}\text { Corneal endothelium } \\
\text { wrinkling }\end{array}$ & 20 & 2.3 & 25 & 3.9 & 0.067 \\
\hline Hypopyon & 49 & 5.5 & 42 & 6.5 & 0.419 \\
\hline Fibrinous exudates & 71 & 8.0 & 33 & 5.1 & $0.030^{*}$ \\
\hline Synechiae & 234 & 26.5 & 140 & 21.8 & $0.040^{*}$ \\
\hline
\end{tabular}

${ }^{*} \mathrm{p}<0.05$.

$A A U$, acute anterior uveitis; $A S$, ankylosing spondylitis; HLA, human leucocyte antigen.

There was no difference concerning the percentage of these FFA features between the HLA-B27 ${ }^{+} \mathrm{AS}^{+}$and HLA-B $27^{+} \mathrm{AS}^{-}$group (table 3).

There was no correlation between retinal vascular leakage and gender, onset age, clinical features including fibrinous exudation, corneal endothelium wrinkling, hypopyon and synechiae, complications including complicated cataract, transiently increased IOP, secondary glaucoma, neovascularisation of the iris and hypotony, nor was there any correlation between the FFA findings and IOP, as well as visual acuity.

\section{Complications and visual outcome}

Complications including complicated cataract, transiently increased IOP, secondary glaucoma, neovascularisation of the iris and hypotony were recorded at each visit during follow-up. The most common complication was complicated cataract $(16.3 \%)$, followed by transiently increased IOP secondary to active inflammation in the anterior chamber (7.6\%) and secondary glaucoma (4.3\%).

The percentage of complicated cataract and secondary glaucoma was significantly higher in the HLA-B $27^{+} \mathrm{AS}^{+}$group as compared with the HLA-B27 ${ }^{+} \mathrm{AS}^{-}$group (table 4). No significant
Table 3 Comparison of FFA findings between $\mathrm{HLA}-\mathrm{B} 27^{+} \mathrm{AS}{ }^{+}$group and HLA-B27 ${ }^{+} \mathrm{AS}^{-}$group

\begin{tabular}{|c|c|c|c|c|c|}
\hline & \multicolumn{2}{|l|}{$\begin{array}{l}\mathrm{AS}^{+} \\
(\mathrm{n}=466)\end{array}$} & \multicolumn{2}{|l|}{$\begin{array}{l}\mathrm{AS}^{-} \\
(\mathrm{n}=437)\end{array}$} & \multirow[b]{2}{*}{ p Value } \\
\hline & Eyes $(n)$ & Rate \% & Eyes $(n)$ & Rate $\%$ & \\
\hline Fluorescence leakage & 170 & 36.5 & 185 & 42.3 & 0.077 \\
\hline Cystoid macular oedema & 9 & 1.9 & 6 & 1.4 & 0.602 \\
\hline Optic disc hyperfluorescence & 24 & 5.2 & 25 & 5.7 & 0.769 \\
\hline
\end{tabular}

difference was found in the percentage of other complications between the two groups of patients.

Visual improvement was observed in patients following treatment except those who had a complete opacity of the lens, severe optic nerve atrophy or hypotony. BCVA was monocularly assessed and recorded in LogMAR scores by using the standard logarithmic visual acuity chart. Worse visual outcome as shown by a higher percentage of patients with a BCVA $<0.5$ or a BCVA $<0.05$ was noted in the HLA-B27 $7^{+} \mathrm{AS}^{+}$group as compared with the HLA-B27 $7^{+} \mathrm{AS}^{-}$group, both before and after treatment (table 5). The BCVA at the last visit was negatively correlated with onset age $(\mathrm{r}=-0.334, \mathrm{p}<0.001)$ and the presence of cataract $(r=-0.416, p<0.001)$.

\section{DISCUSSION}

Our uveitis clinic is the largest tertiary uveitis centre in China, and to our knowledge, this study represents the largest retrospective epidemiological survey of HLA-B27 ${ }^{+}$AAU in mainland China. Among the patients with AAU being referred to our clinic during the last 8 years, it appeared that HLA-B $27^{+}$AAU was the most common entity with a percentage of $61.5 \%$ among all AAU patients. Since we focused on the question how the occurrence of AS affects the manifestations of HLA-B27 $7^{+}$AAU, the HLA-B27 ${ }^{+}$ AAU patients with other seronegative spondyloarthropathies such as inflammatory bowel disease, psoriatic arthritis, reactive arthritis and sarcoidosis were excluded from this study. The result revealed that HLA-B $27^{+} \mathrm{AS}^{+}$AAU patients showed
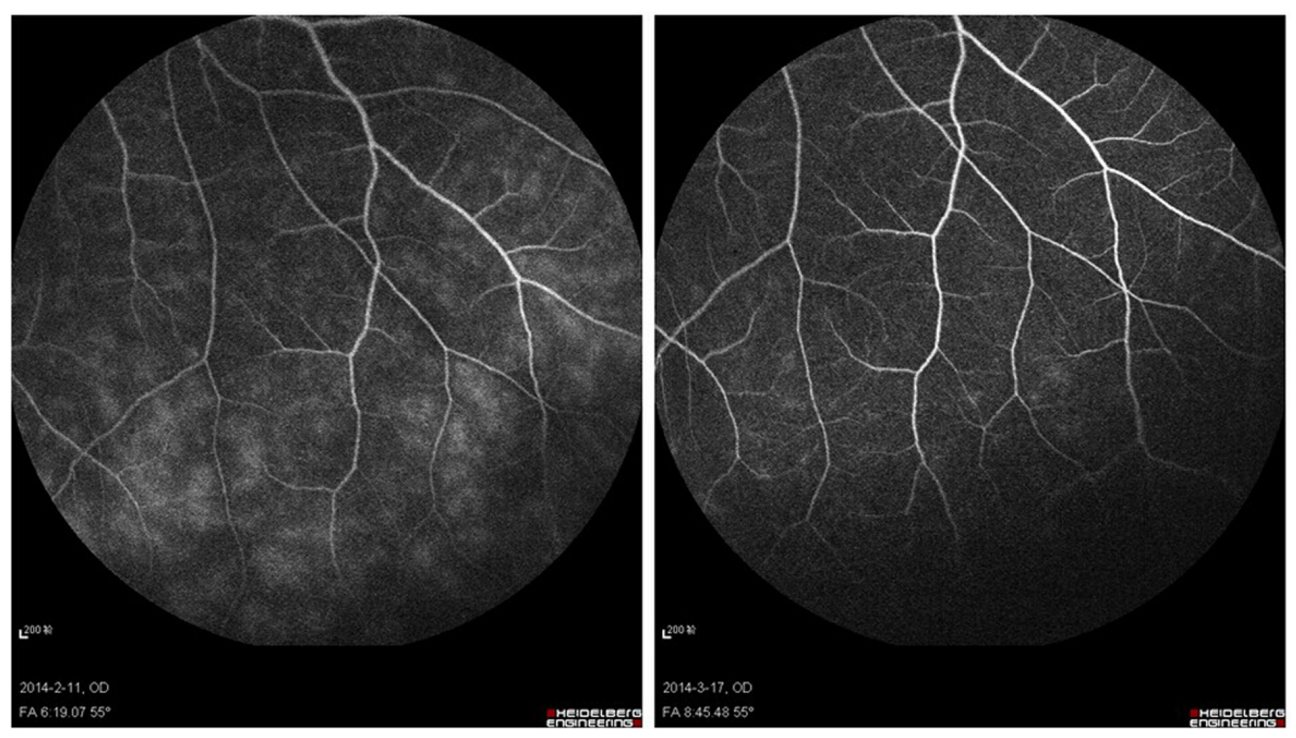

Figure 1 Retina vascular fluorescence leakage disclosed by FFA in a 30-year-old male patient of HLA-B27 ${ }^{+} \mathrm{AS}^{-}$group. Left: The result obtained at first visit, 8 days after AAU onset showed diffuse vascular fluorescence leakage on the peripheral retinal. Right: The result obtained 42 days after AAU onset showed that the vascular fluorescence leakage observed the first visit almost disappeared. AAU, acute anterior uveitis; AS, ankylosing spondylitis; FFA, fundus fluorescence angiography; HLA, human leucocyte antigen. 
Table 4 Comparison of complications between $\mathrm{HLA}-\mathrm{B} 27^{+} \mathrm{AS}^{+}$group and $\mathrm{HLA}-\mathrm{B} 27^{+} \mathrm{AS}^{-}$group

\begin{tabular}{|c|c|c|c|c|c|}
\hline \multirow[b]{2}{*}{ Complication } & \multicolumn{2}{|l|}{$\begin{array}{l}\mathrm{AS}^{+} \\
(\mathrm{n}=883)\end{array}$} & \multicolumn{2}{|l|}{$\begin{array}{l}\mathrm{AS}^{-} \\
(\mathrm{n}=642)\end{array}$} & \multirow[b]{2}{*}{$p$ Value } \\
\hline & Eyes ( $n$ ) & Rate $\%$ & Eyes $(n)$ & Rate \% & \\
\hline Complicated cataract & 164 & 18.6 & 86 & 13.4 & $0.008^{* *}$ \\
\hline Transiently increased IOP & 64 & 7.2 & 52 & 8.1 & 0.558 \\
\hline Secondary glaucoma & 49 & 5.5 & 16 & 2.5 & $0.004^{* *}$ \\
\hline Neovascularisation of iris & 12 & 1.4 & 9 & 1.4 & 0.943 \\
\hline Hypotony & 1 & 0.1 & 1 & 0.2 & 0.821 \\
\hline
\end{tabular}

${ }^{*} p<0.05,{ }^{* *} 0.001 \leq p \leq 0.01,{ }^{* * *} p<0.001$

AS, ankylosing spondylitis; HLA, human leucocyte antigen; IOP, intraocular pressure.

a higher percentage of males and bilateral/alternating involvement, more likely to be accompanied with fibrinous exudation, synechiae, complicated cataract and secondary glaucoma, as well as a worse visual outcome as compared with $\mathrm{HLA}-\mathrm{B} 27^{+} \mathrm{AS}^{-}$ AAU patients. A novel finding of our study was the observation that retinal vascular leakage occurred in approximately $40 \%$ of the HLA-B27 ${ }^{+}$AAU patients.

The higher prevalence of males in $\mathrm{HLA}-\mathrm{B} 27^{+} \mathrm{AS}^{+}$AAU patients is in agreement with earlier studies. ${ }^{16}{ }^{17}$ A larger degree of bilateral/alternating involvement in $\mathrm{HLA}-\mathrm{B} 27^{+} \mathrm{AS}^{+}$ AAU patients, is also consistent with earlier studies. ${ }^{18} \mathrm{~A}$ more intense inflammatory reaction as evidenced by the appearance of fibrinous exudates in the anterior chamber in HLA-B $27^{+} \mathrm{AS}^{+}$ AAU patients as compared with the HLA-B27 ${ }^{+} \mathrm{AS}^{-}$AAU patients as found in our study, has also been noted earlier by others. ${ }^{19}$

It has been documented that AAU may affect the posterior segment, although there are no apparent retinal or choroidoretinal lesions. ${ }^{20}$ OCT analysis has shown that eyes with acute anterior uveitis had thickened maculae and peripapillary retinal nerve fibre layer as compared with controls. ${ }^{21}$ Macular thickness was closely correlated with the inflammation in anterior uveitis patients with spondyloarthropathy. ${ }^{22}$ Shulman et $a l^{21}$ assumed that this might be associated with a breakdown in the blood-aqueous and blood-retinal barriers and an increase in vascular permeability. These earlier investigations all focused on the posterior pole but did not assess the peripheral retina. FFA has been widely used to evaluate abnormalities of the retina, retinal blood vessels and optic nerve. Using this technique, it was interesting to note that a number of abnormalities including retinal vascular leakage, cystoid macular oedema and optic disc hyperfluorescence were observed in HLA-B $27^{+}$AAU patients, and as mentioned above, retinal vascular fluorescence leakage was not uncommon in these patients. It is not clear whether this abnormality is caused by a direct retinal involvement of the

Table 5 Comparison of visual outcome between $\mathrm{HLA}-\mathrm{B} 27^{+} \mathrm{AS}^{+}$ group and $\mathrm{HLA}-\mathrm{B} 27^{+} \mathrm{AS}^{-}$group

\begin{tabular}{|c|c|c|c|c|c|}
\hline \multirow[b]{2}{*}{ Visual outcome } & \multicolumn{2}{|l|}{$\begin{array}{l}\mathrm{AS}^{+} \\
(\mathrm{n}=883)\end{array}$} & \multicolumn{2}{|l|}{$\begin{array}{l}\mathrm{AS}^{-} \\
(\mathrm{n}=642)\end{array}$} & \multirow[b]{2}{*}{ p Value } \\
\hline & Eyes $(n)$ & Rate $\%$ & Eyes (n) & Rate $\%$ & \\
\hline BCVA of first visit $<0.5$ & 367 & 41.6 & 224 & 34.9 & $0.009 * *$ \\
\hline BCVA of first visit $<0.05$ & 89 & 10.1 & 36 & 5.6 & $0.002^{* *}$ \\
\hline BCVA of last visit $<0.5$ & 168 & 19.0 & 87 & 13.6 & $0.005^{* *}$ \\
\hline BCVA of last visit $<0.05$ & 42 & 4.8 & 14 & 2.2 & $0.008 * *$ \\
\hline
\end{tabular}

${ }^{*} \mathrm{p}<0.05,{ }^{* *} 0.001 \leq \mathrm{p} \leq 0.01,{ }^{* * *} \mathrm{p}<0.001$.

$A A U$, acute anterior uveitis; $A S$, ankylosing spondylitis; $B C V A$, best corrected visual acuity; HLA, human leucocyte antigen. inflammation or whether it is caused by an effect of inflammatory mediators released from the anterior segment. Fortunately, the abnormalities including retinal vascular leakage, cystoid macular oedema and optic disc hyperfluorescence disclosed by FFA were not correlated to visual outcome, and resolved immediately following the disappearance of anterior segment inflammation without any complications. FFA is therefore not crucial in guiding the ophthalmologist concerning the further management of these patients and merely indicates posterior segment involvement. Of interest is the observation that endotoxin-induced uveitis, which serves as an experimental animal model of AAU, is also associated with posterior segment involvement as evidenced by retinal macrophage infiltration. ${ }^{23}$

It has been reported that AS or the association with HLA-B27 may influence the occurrence of complications and visual prognosis in AAU. ${ }^{19} 2425$ Complications including complicated cataract, ocular hypertension, secondary glaucoma and cystoid macular oedema were less common in HLA-B27 ${ }^{+}$AAU patients as compared with HLA-B27- AAU patients. ${ }^{19}{ }^{25}$ However, no significant difference in complications or visual outcome were reported between HLA-B2 $7^{+}$AAU patients with or without AS. ${ }^{26}$ In general, the HLA-B27 ${ }^{+}$patients had a better visual prognosis as compared with uveitis patients with Vogt-Koyanagi-Harada syndrome $^{27}$ or Behcet's disease. ${ }^{28}$ A poorer visual outcome was found in patients with AS, which is probably due to the higher frequency of complicated cataract.

There are several limitations in our study. It is a retrospective study and some of the results, such as the observation of the high degree of retinal vascular leakage, should be confirmed in prospective studies. Although the FFA findings have not yet changed our management of these patients, further study is needed to investigate the prognosis of patients with serious retinal vascular leakage and whether they would benefit from earlier and more aggressive treatment. In the 581 AS patients with AAU, only 42 patients were treated by specialists in rheumatology or orthopaedics with tumour necrosis factor (TNF) inhibitors. As the durations and dosages of the treatment were different, it is not clear whether the TNF inhibitors could influence the visual prognosis and FFA findings in these patients. It is also not clear whether these retinal findings are a typical manifestation in Chinese patients and comparison with patients from other ethnic backgrounds is needed to clarify this issue. A variety of treatment regimens including corticosteroids, non-steroidal anti-inflammatory drugs, immunosuppressive agents and biological agents have been used for the treatment of our AS patients and comparison with patients without AS who received a milder treatment may therefore be a controversial issue. Despite the more aggressive treatment, the AS patients did worse and we would like to conclude that the occurrence of AS in our AAU patients is a manifestation of a more serious form of the same disease, although further studies are needed to support this hypothesis.

In conclusion, our study characterised the clinical features of HLA-B $27^{+}$AAU with or without AS based on a large cohort of Chinese patients. Interestingly, our study showed that retinal vascular involvement was a relatively common manifestation in these patients. Our results also demonstrated a male predominance, bilateral/alternating involvement and poorer visual prognosis in HLA-B $27^{+}$AAU patients with AS.

Acknowledgements We appreciate Professor Zhenming Hu, Professor Wei Huang from the orthopaedics department and Professor Rongheng Li from the rheumatology department of the First Affiliated Hospital of Chongqing Medical University for their assistance in the diagnosis and treatment of ankylosing spondylitis. 
Contributors PY and WW designed the study and analysed the data. LD, QZ, JQ LL, CW and LW collected the data. PY and WW drafted the manuscript. AK helped revise the manuscript. All authors reviewed the manuscript.

Funding This work was supported in part by Clinic Key Project of Ministry of Health (201002019), Chongqing Key Laboratory of Ophthalmology (CSTC, 2008CA5003), National Key Clinical Specialties Construction Program of China, and Key Project of Health Bureau of Chongqing (2012-1-003).

Competing interests None declared.

Patient consent Detail has been removed from this case description/these case descriptions to ensure anonymity. The editors and reviewers have seen the detailed information available and are satisfied that the information backs up the case the authors are making.

Provenance and peer review Not commissioned; externally peer reviewed.

Open Access This is an Open Access article distributed in accordance with the Creative Commons Attribution Non Commercial (CC BY-NC 4.0) license, which permits others to distribute, remix, adapt, build upon this work non-commercially, and license their derivative works on different terms, provided the original work is properly cited and the use is non-commercial. See: http://creativecommons.org/ licenses/by-nc/4.0/

(c) Article author(s) (or their employer(s) unless otherwise stated in the text of the article) 2018. All rights reserved. No commercial use is permitted unless otherwise expressly granted.

\section{REFERENCES}

1 Brewerton D, Caftrey M, Nicholls A. Acute anterior uveitis and HLA B27. Lancet 1973;2:994-6.

2 McCannel CA, Holland GN, Helm CJ, et al. Causes of uveitis in the general practice of ophthalmology. UCLA Community-Based Uveitis Study Group. Am J Ophthalmol 1996;121:35-46.

3 Chang JH, McCluskey PJ, Wakefield D. Acute anterior uveitis and HLA-B27. Surv Ophthalmol 2005:50:364-88.

4 Accorinti M, lannetti L, Liverani M, et al. Clinical features and prognosis of HLA B27-associated acute anterior uveitis in an italian patient population. Ocul Immunol Inflamm 2010;18:91-6.

5 Chung YM, Yeh TS, Liu JH. Endogenous uveitis in chinese--an analysis of 240 cases in a uveitis clinic. Jpn J Ophthalmol 1988;32:64-9.

6 Yang $\mathrm{P}$, Zhang Z, Zhou H, et al. Clinical patterns and characteristics of uveitis in a tertiary center for uveitis in China. Curr Eye Res 2005;30:943-8.

7 Monnet D, Breban M, Hudry C, et al. Ophthalmic findings and frequency of extraocular manifestations in patients with HLA-B27 uveitis: a study of 175 cases. Ophthalmology 2004;111:802-9.

8 Paiva ES, Macaluso DC, Edwards A, et al. Characterisation of uveitis in patients with psoriatic arthritis. Ann Rheum Dis 2000;59:67-70.

9 Beckingsale AB, Davies J, Gibson JM, et al. Acute anterior uveitis, ankylosing spondylitis, back pain, and HLA-B27. Br J Ophthalmol 1984;68:741-5.
10 Bañares A, Hernández-García C, Fernández-Gutiérrez B, et al. Eye involvement in the spondyloarthropathies. Rheum Dis Clin North Am 1998;24:771-84.

11 Hoeksema L, Los LI. Visual prognosis and ocular complications in herpetic versus HLA-B27- or ankylosing Spondylitis-associated Anterior Uveitis. Ocul Immunol Inflamm 2016;24:1-11.

12 Jabs D, Nussenblatt R, Rosenbaum J. The Standardization of Uveitis Nomenclature (SUN) Working Group. Standardization of uveitis nomenclature for reporting clinical data. Results of the First International Workshop. Am J Ophthalmol 2005;140:509-16.

13 van der Linden S, Valkenburg HA, Cats A. Evaluation of diagnostic criteria for ankylosing spondylitis. A proposal for modification of the New York criteria. Arthritis Rheum 1984;27:361-8.

14 Garrett S, Jenkinson T, Kennedy LG, et al. A new approach to defining disease status in ankylosing spondylitis: the Bath Ankylosing Spondylitis Disease Activity Index. J Rheumatol 1994:21:2286-91.

15 Calin A, Garrett S, Whitelock H, et al. A new approach to defining functional ability in ankylosing spondylitis: the development of the Bath Ankylosing Spondylitis functional index. J Rheumatol 1994;21:2281-5.

16 Chung YM, Liao HT, Lin KC, et al. Prevalence of spondyloarthritis in 504 chinese patients with HLA-B27-associated acute anterior uveitis. Scand J Rheumatol 2009;38:84-90.

17 Kim TJ, Na KS, Lee HJ, et al. HLA-B27 homozygosity has no influence on clinical manifestations and functional disability in ankylosing spondylitis. Clin Exp Rheumatol 2009:27:574-9.

18 Cantini F, Nannini C, Cassarà E, et al. Uveitis in Spondyloarthritis: an overview. J Rheumatol Supp/ 2015:93:27-9.

19 Pathanapitoon K, Suksomboon S, Kunavisarut P, et al. HLA-B27-associated acute anterior uveitis in the University Referral Centre in North Thailand: clinical presentation and visual prognosis. Br J Ophthalmol 2006;90:1448-50.

20 Moschos MM. Subclinical macular edema detected by Spectral-domain Optical Coherence Tomography (SD-OCT) in HLA-B27 positive Anterior Uveitis. Med Hypothesis Discov Innov Ophthalmol 2014;3:123-6.

21 Shulman S, Goldenberg D, Habot-Wilner Z, et al. Optical coherence tomography characteristics of eyes with acute anterior uveitis. Isr Med Assoc J 2012;14:543-6.

22 Traill A, Stawell R, Hall A, et al. Macular thickening in acute anterior uveitis. Ophthalmology 2007;114:402.

23 Yang P, Smith JR, Damodar KS, et al. Visualization of cell death in vivo during murine endotoxin-induced uveitis. Invest Ophthalmol Vis Sci 2003;44:1993-7.

24 Linssen A, Meenken C. Outcomes of HLA-B27-positive and HLA-B27-negative acute anterior uveitis. Am J Ophthalmol 1995;120:351-61.

25 Karaconji T, Maconochie Z, McCluskey P. Acute anterior uveitis in Sydney. Ocul Immunol Inflamm 2013;21:108-14

26 Tseng ST, Yao TC, Huang JL, et al. Clinical manifestations in uveitis patients with and without rheumatic disease in a Chinese population in Taiwan. J Microbiol Immunol Infect 2017; 50:798-804.

27 Yang P, Ren Y, Li B, et al. Clinical characteristics of Vogt-Koyanagi-Harada syndrome in chinese patients. Ophthalmology 2007;114:606-14.

28 Yang P, Fang W, Meng Q, et al. Clinical features of chinese patients with Behçet's disease. Ophthalmology 2008;115:312-8. 